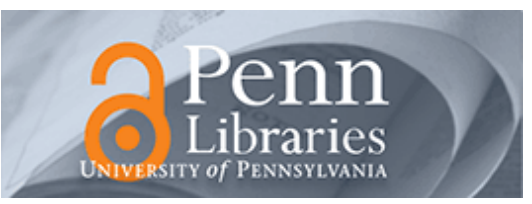

University of Pennsylvania

ScholarlyCommons

\title{
$5-2015$
}

\section{Establishing a Clinically Relevant Large Animal Model Platform for TBI Therapy Development: Using Cyclosporin A as a Case Study}

\author{
Susan S. Margulies \\ University of Pennsylvania, margulies@seas.upenn.edu \\ Todd Kilbaugh \\ University of Pennsylvania, kilbaugh@chop.edu \\ Sarah Sullivan \\ Colin Smtih \\ Kathleen J. Propert
}

See next page for additional authors

Follow this and additional works at: https://repository.upenn.edu/be_papers

Part of the Biomedical Engineering and Bioengineering Commons

\section{Recommended Citation}

Margulies, S. S., Kilbaugh, T., Sullivan, S., Smtih, C., Propert, K. J., Byro, M., Saliga, K., Costine, B. A., \& Duhaime, A. (2015). Establishing a Clinically Relevant Large Animal Model Platform for TBI Therapy Development: Using Cyclosporin A as a Case Study. Brain Pathology, 25 (3), 289-303. http://dx.doi.org/ 10.1111/bpa. 12247

This paper is posted at ScholarlyCommons. https://repository.upenn.edu/be_papers/215

For more information, please contact repository@pobox.upenn.edu. 


\title{
Establishing a Clinically Relevant Large Animal Model Platform for TBI Therapy Development: Using Cyclosporin A as a Case Study
}

\begin{abstract}
We have developed the first immature large animal translational treatment trial of a pharmacologic intervention for traumatic brain injury (TBI) in children. The preclinical trial design includes multiple doses of the intervention in two different injury types (focal and diffuse) to bracket the range seen in clinical injury and uses two post-TBI delays to drug administration. Cyclosporin A (CsA) was used as a case study in our first implementation of the platform because of its success in multiple preclinical adult rodent TBI models and its current use in children for other indications. Tier 1 of the therapy development platform assessed the short-term treatment efficacy after $24 \mathrm{~h}$ of agent administration. Positive responses to treatment were compared with injured controls using an objective effect threshold established prior to the study. Effective CsA doses were identified to study in Tier 2. In the Tier 2 paradigm, agent is administered in a porcine intensive care unit utilizing neurological monitoring and clinically relevant management strategies, and intervention efficacy is defined as improvement in longer term behavioral endpoints above untreated injured animals. In summary, this innovative large animal preclinical study design can be applied to future evaluations of other agents that promote recovery or repair after TBI.
\end{abstract}

\section{Keywords}

brain injury, pediatric, preclinical trials, treatment, TBI

\section{Disciplines}

Biomedical Engineering and Bioengineering | Engineering

\section{Author(s)}

Susan S. Margulies, Todd Kilbaugh, Sarah Sullivan, Colin Smtih, Kathleen J. Propert, Melissa Byro, Kristen Saliga, Beth A. Costine, and Ann-Christine Duhaime 


\title{
Establishing a Clinically Relevant Large Animal Model Platform for TBI Therapy Development: Using Cyclosporin A as a Case Study
}

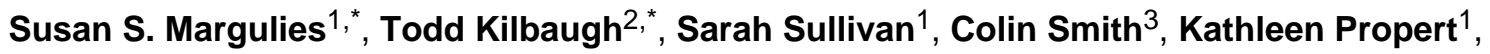 \\ Melissa Byro ${ }^{1}$, Kristen Saliga ${ }^{4}$, Beth A. Costine ${ }^{4}$, and Ann-Christine Duhaime ${ }^{4}$ \\ ${ }^{1}$ University of Pennsylvania, Philadelphia, PA. ${ }^{2}$ Children's Hospital of Philadelphia, Philadelphia, \\ PA. ${ }^{3}$ Edinburgh University, Edinburgh, Scotland. ${ }^{4}$ Massachusetts General Hospital, Harvard \\ University, Boston, MA.
}

\begin{abstract}
We have developed the first immature large animal translational treatment trial of a pharmacologic intervention for traumatic brain injury (TBI) in children. The preclinical trial design includes multiple doses of the intervention in two different injury types (focal and diffuse) to bracket the range seen in clinical injury and uses two post-TBI delays to drug administration. Cyclosporin A (CsA) was used as a case study in our first implementation of the platform because of its success in multiple preclinical adult rodent TBI models and its current use in children for other indications. Tier 1 of the therapy development platform assessed the short-term treatment efficacy after $24 \mathrm{~h}$ of agent administration. Positive responses to treatment were compared with injured controls using an objective effect threshold established prior to the study. Effective CsA doses were identified to study in Tier 2. In the Tier 2 paradigm, agent is administered in a porcine intensive care unit utilizing neurological monitoring and clinically relevant management strategies, and intervention efficacy is defined as improvement in longer term behavioral endpoints above untreated injured animals. In summary, this innovative large animal preclinical study design can be applied to future evaluations of other agents that promote recovery or repair after TBI.
\end{abstract}

\section{Keywords}

brain injury; pediatric; preclinical trials; treatment; TBI

\section{INTRODUCTION}

Head injury is the leading cause of death and disability in children (38), and unfortunately there are only general management guidelines (3). While only a modest number of pediatric

\footnotetext{
(C) 2015 International Society of Neuropathology

Corresponding Author: Susan S. Margulies, PhD, Department of Bioengineering, University of Pennsylvania, 210 S. 33rd. St., 240 Skirkanich Hall, Philadelphia, PA 19104-6321 (margulies@seas.upenn.edu).

${ }^{*}$ An asterisk designates joint first authorship.
} 
clinical trials for traumatic brain injury (TBI) have been conducted $(1,39,42,61,66,93)$, nearly all pediatric trials and over 100 adult TBI trials $(9,14,56,71)$ have failed to show significant neuroprotective benefits of any pharmacologic therapy.

While general care principles have improved outcomes, specific neuroprotective interventions are extremely limited. The heterogeneity of TBI, with distinct pathophysiologies and mechanisms associated with contusions, hemorrhages and diffuse axonal injuries, added to a panoply of variable host factors, likely contributes to the recurrent failure of large head injury treatment trials $(60,74)$. In children, the immature brain response to each type of TBI changes rapidly during development from infancy through adolescence $(7,20,22,68)$. Therefore, the development of head injury therapies for acute brain injury in children requires appropriate immature animal models in which to test potential treatments. Rodents are limited as models for human children because of marked differences in maturation, morphology and injury mechanisms (17). Therefore, treatments developed in adult rodents might be ineffective, or even contraindicated, in human children.

Motivated by the need for appropriate immature animal models, our collaborating laboratories have developed, well-characterized and thoroughly tested immature swine models for TBI research for more than 15 years $(20,21,24,29,51,59,68,69,77,81)$. These models mimic the spectrum of TBI observed in infants and children. The purely inertial (nonimpact) rotation model creates diffuse axonal injury and subarachnoid hemorrhage, and the purely focal model creates a localized cortical and subcortical contusion (Figure 1). By using both models, we have characterized the response of the immature brain at the two endpoints of the focal-to-diffuse and cortical surface to axonal white matter injury spectrum. To characterize changing injury responses in the maturing brain, we have studied different developmental stages, infancy through adolescence, in both piglet models. From these studies, we have determined that the 1-month age ("toddler") piglet has unique characteristics that reflect pathophysiology similar to the preschool and early childhood stage in human children. At this age, cerebral blood flow (CBF) is maximal, brain swelling is the most pronounced, and other injury responses are similar to the unique findings seen in young human children $(19,20,23)$.

We have designed an innovative, state-of-the-art preclinical study platform to create a translational bridge between basic science discoveries in the rodent and randomized control trials for TBI in children. Our preclinical study was led by a collaborative team of pediatric TBI experts able to measure the pharmacokinetics (PK) and the short- and long-term pharmacodynamic response to treatment for TBI. To duplicate the human clinical setting, the platform incorporates the best contemporary pediatric neurocritical care management and monitoring strategies to enhance translation to clinical trials. Using our wellcharacterized, reproducible and clinically relevant immature porcine TBI models, we employed well-developed assessments of physiologic, biochemical and cognitive/behavioral outcomes that we believe surpass rodent models for their fidelity to children to determine the optimal evidence-based dosing strategy for a future large randomized controlled trial for treatments in children with TBI. 
Because mitochondria play a critical role in many primary and secondary pathologic pathways in TBI, cyclosporin A (CsA), which inhibits opening of the mitochondrial permeability transition pore, was used as a case study in our first implementation of the platform. Because of its safety profile in humans, pleiotropic effects and success in multiple preclinical adult rodent TBI models, CsA was selected for its multifaceted potential as an effective therapy for pediatric TBI. Because CsA was also off-patent and already in use in children for other indications, the results of the preclinical therapy development plan could be translated rapidly and inexpensively to clinical trial.

\section{METHODS}

\section{Animal model selection}

We have used our established immature porcine TBI models, controlled cortical impact (CCI) and rapid nonimpact rotation (RNR), to produce the spectrum of pathophysiology seen in mild to severe TBI in the child, from infancy to early adolescence. The CCI (Figure 1) model utilizes a skull-mounted, spring-loaded blunt indentation device to create a rapid (4 ms) displacement of the cortical surface, producing a focal cortical contusion, with underlying white matter damage, decreased CBF and somatosensory dysfunction (19-21). This model was designed to produce a specific injury type, focal contusion, which is one of the most common types of brain injury in children and results most often from falls, recreational and sports injuries, and vehicular trauma $(34,46,82)$. Because the displacement of the cortex is coupled to the skull, this model produces no inertial motion of the head itself, and in this way embodies a purely focal injury mechanism. The model creates a welldefined cortical and subcortical contusion with variable hemorrhage which evolves over time, is associated with regional brain swelling and changes in blood flow, and can be measured by histology or imaging. The contusion is placed in the rostral gyrus, which is the somatosensory cortex subserving snout sensation. The injury is not associated with immediate changes in consciousness or gross motor deficits. At the other end of the injury spectrum, to create a purely inertial injury, the RNR model produces a high magnitude acceleration/deceleration, similar to that experienced in motor vehicle or high velocity trauma, but with no impact event, and utilizes a single rapid (12-20 ms), sagittal head rotation (Figure 1). This injury mechanism produces unconsciousness, sustained cognitive dysfunction, bilateral diffuse axonal and hemorrhagic injury; and marked decreases in global $\mathrm{CBF}$, brain tissue oxygen content $\left(\mathrm{PbtO}_{2}\right)$ and alterations in mitochondrial function $(24,29$, 68). Our goal was to inform a clinical study with injury-specific optimal dosing strategies by evaluating treatment effectiveness over the spectrum of injury types.

Based on our prior studies of brain development and age-dependent injury responses, the 4week-old piglet is similar to human children at the toddler/preschool age $(17,18,20,23$, 57). Age-specific responses are attributable to maturational differences in brain mass, stiffness, deformation tolerance and biological responses. The toddler age in the child was chosen for our first test of this large animal model platform for therapeutic development because it is an age with physiologically important and maximal developmental differences in myelination, apoptotic pathways, glutamatergic receptor mechanisms, synaptogenesis and 
CBF responses compared with adults. The toddler piglet mimics the range of measureable post-TBI morbidity seen in the human toddler and young child.

\section{Therapy development plan overview}

Recent workshops of the TBI and stroke scientific communities have examined why agents with preclinical therapeutic efficacy have failed to translate to clinical success $(15,27,36$, 50). They concluded that agents should be tested in multiple species using clinically relevant outcomes, short-term and long-term endpoints, histological and functional metrics, and include clinical management strategies (45). We conducted our therapy development studies across multiple laboratories, while including current pediatric TBI critical care management protocols, clinically relevant physiological monitoring, and realistic injury-to-treatment intervals of 1 and $6 \mathrm{~h}$ after TBI, to mimic urban and rural time to implantation of therapy. Treatments were administered intravenously (IV) for $24 \mathrm{~h}$ via an ambulatory wireless programmable infusion pump, delivering a loading dose followed by continuous infusion to achieve target concentrations rapidly and to sustain exposure, similar to current clinical studies for adult TBI (54). The platform employs a two-tiered funnel approach: focusing first on multiple short-term ( $24 \mathrm{~h}$ ) endpoints-histological and cellular bioenergetic measures-to identify dosing strategies with a reproducible, robust efficacy, followed by studies evaluating sustained cognitive benefits. Importantly, the platform includes obtaining microdialysate samples and blood for serum biomarkers, currently unconfirmed clinical intensive care readouts in children, and in future secondary analyses we plan to correlate them with direct measures (mitochondrial function and pathology) and neurofunctional behavior. These analyses, which are an important feature of our preclinical development and optimization plan, will accelerate the translation to a rational clinical trial design, informing the monitoring and management strategy, as well as inclusion criteria. Finally, although we recognized the potential for inflation of type I error through the multiple experiments utilized in the platform, the use of efficient factorial design in combination with the overall sequenced study design was created to optimize identification of promising treatments in an important clinical area with no effective pharmacologic interventions. In summary, this state-of-the-art, innovative preclinical study design will be applied to future evaluations of other agents that promote recovery or repair after TBI, other ages, combination therapies and other types of TBI.

In the first tier of our Therapy Development Plan, we evaluated the tolerability and efficacy of the therapeutic agent to identify two promising doses for each injury type (diffuse, focal) and post-TBI treatment start times ( 1 and $6 \mathrm{~h}$ ) based on terminal endpoints of neuroprotective effectiveness at $24 \mathrm{~h}$. In the second tier, the optimum dose for each injury type and start time with the best 6-day cognitive outcomes is identified. In Tier 1, for each start time and injury type, both the most effective dose and the lowest dose that achieve a significant effect advance to Tier 2 for further study. In Tier 2, not yet conducted, these two doses for each start time and injury type are assessed for their efficacy in neurocognitive outcomes, measured 6 days after injury, to identify the optimal dosing strategy to evaluate in clinical trials. Later studies will identify PK, toxicology responses and sex-specific cognitive recoveries to the therapeutic dosing strategy and injury type that showed the best cognitive recovery in Tier 2. 
As a proof-of-translation study, we used the immature large animal model platform as a preclinical study to evaluate the effectiveness of agents with a demonstrated track record of efficacy in adult rodent focal neural injury models and an established safety profile in children. Additional considerations may also be that the agent is in therapeutic use or clinical trials for other indications in adults and children, enhancing rapid translation to a clinical trial. We selected CsA as our case study, and our rationale for agent selection is discussed in the next section.

\section{Agent selection}

TBI processes, including contusion, diffuse or focal axonal injury, hematomas, and subarachnoid hemorrhage $(1,2,58,74)$, can initiate ischemia, edema, inflammation, tissue shifts and brain herniation (9). Subsequent cellular and molecular responses progress over minutes, hours and days to mediate progressive cellular damage $(50,52,65,67,76,95)$, decreased CBF and mitochondrial disturbances (35, 52, 65, 80, 91, 97). A growing body of literature suggests that mitochondria play a key role in many pathologic pathways in neurodegenerative disorders, focal/global ischemia and trauma $(48,73,75)$. Mitochondrial dysfunction leads to energy imbalance, ionic imbalance, release of cytochrome c, proapoptotic events, mitochondrial swelling and reduced brain ATP levels (86).

Following pediatric TBI, mitochondrial dysfunction is involved in excitotoxicity, oxidative stress, metabolic perturbations and, ultimately, cell death (73). It is difficult to extrapolate adult TBI data to pediatric models because critical mitochondrial characteristics such as the number and density of complexes of the electron transfer chain, antioxidant enzyme activity and content, and lipid content are very different between young and adult animals $(8,16)$. To our knowledge, no investigators have tested potential mitochondria-targeted neuroprotective therapeutic strategies in a large animal model of pediatric TBI.

CsA inhibits progressive mitochondrial dysfunction by stabilizing the mitochondrial transition pore (87). Preclinical TBI and ischemia CsA studies (mostly in rodents) have demonstrated neuroprotection using immunohistochemistry, isolated mitochondrial preparations and behavioral tests $(6,25,28,30,37,47,78,83,87)$. The advantages of CsA are that it is FDA approved, off-patent, inexpensive and thought to have well-described safety and dosing profiles. In January 2015, there were 109 open clinical trials using CsA in children for indications other than brain injury. In a TBI Phase I clinical trial in adults, CsA satisfied a broad range of safety parameters (54), but no Phase II or III trial is in progress (53). Although CsA has delayed brain penetration and its chronic usage adversely impacts the immune system, we may avoid similar immunological concerns when CsA is limited to a short period, such as for acute TBI treatment. In summary, because of its safety profile in humans, multimodal pharmacological effects, and success in multiple preclinical rodent models of brain injury, CsA has potential as a therapeutic agent for neuroprotection in children after TBI. We hypothesized that CsA would inhibit mitochondrial dysfunction and decrease axonal and neuronal injury within $24 \mathrm{~h}$ after head trauma, and would promote longer term neurological functional recovery. Our objective was to obtain data on the PK and pharmacodynamics of CsA in two established immature large animal preclinical models to provide evidence-based dosing guidance for a future large randomized controlled trial for 
CsA in children. Our central hypothesis is that safe, therapeutic concentrations of CsA could improve outcomes for children who have sustained TBI.

\section{Dosing rationale-allometric scaling across species}

Tier 1 dose selection is based on published rodent neuro-injury $(6,55,87)$, our previous data in the infant piglet (44), published dosing guidelines, and Phase I-IV clinical trials. Rather than scaling the dose across species normalizing by body weight, more recent PK literature advocates the extrapolation of rat dose to pig and human doses be performed through normalization to body surface area using the $K_{\mathrm{m}}$ factor and the following formula: dose for animal $\mathrm{x}$ in $\mathrm{mg} / \mathrm{kg}$ equals the dose in animal y in $\mathrm{mg} / \mathrm{kg}$ times $\left(\mathrm{K}_{\mathrm{my}} / K_{\mathrm{mx}}\right)(70)$, where the estimated $K_{\mathrm{m}}$ for the rat, 4-week-old piglet, human child and adult are 6, 20, 25 and 37, respectively. First, CsA was studied in an adult rat model of focal TBI, and a U-shaped dose-response was observed $(6,55,87)$, such that the most beneficial dose was determined to be $20 \mathrm{mg} / \mathrm{kg} /$ day divided in two $10 \mathrm{mg} / \mathrm{kg}$ doses (87). Using relevant allometric $K_{\mathrm{m}}$, this optimal rat dose translates into $6 \mathrm{mg} / \mathrm{kg} / \mathrm{day}$ in the pig and $3.3 \mathrm{mg} / \mathrm{kg}$ day in the human adult. Second, CsA is being used clinically in the human in doses between 5 and 21 $\mathrm{mg} / \mathrm{kg} / \mathrm{day}(32,49,54)$, which translate into "safe" doses of 9.25 and $39 \mathrm{mg} / \mathrm{kg} /$ day in the pig and 30 and $126 \mathrm{mg} / \mathrm{kg} / \mathrm{day}$ in the rat. Third, in the infant piglet, we have conducted pilot studies ( $\mathrm{N}=4-5 /$ group$)$ and found that an IV bolus of CsA $(20 \mathrm{mg} / \mathrm{kg}$ ) given $5 \mathrm{~min}$ and again at $12 \mathrm{~h}$ after RNR TBI (totaling $40 \mathrm{mg} / \mathrm{kg} /$ day) markedly reduced lesion volume and lactate-pyruvate ratios $24 \mathrm{~h}$ after TBI, with increased CBF and mitochondrial respiratory control ratio (RCR) in hippocampus, olfactory, cortex and cerebellum, compared with saline-treated injured piglets. Given the U-shaped pharmacodynamics of CsA (85), the toxicity data from humans, and effective doses in rat and piglet, we investigated four CsA doses higher and lower than our infant piglet studies: 10, 20, 40 and $60 \mathrm{mg} / \mathrm{kg} / \mathrm{day}$ for Tier 1 optimization studies, given toxicity data in humans and doses deemed effective in the rat and piglet. Based upon human TBI clinical trials, rodent CCI and porcine RNR studies, treatment duration was $24 \mathrm{~h}$ for all tiers. The half-life of CsA is long, approximately $18 \mathrm{~h}$ in newborn pigs and $7 \mathrm{~h}$ in mature pigs (92), and steady state exposure cannot be reached during a 24-h treatment duration. A rapid loading dose can achieve therapeutic concentrations faster, but plasma CsA levels may exceed tolerable concentrations $(26,84)$. Renal tolerance limits in the pig are $9 \mathrm{mg} / \mathrm{kg}$ IV over $1 \mathrm{~h}$ (13), but higher loading doses have not been studied and may be tolerated. In summary, based on CsA half-life, published CsA pig clearance rates and tolerance, and the current human clinical trials employing continuous IV infusion, the 24-h dose strategy was to be divided into a 1-h IV loading infusion to reach target concentrations rapidly, with the remainder delivered in a 23-h continuous IV infusion for sustained exposure, with a ratio (in $\mathrm{mg} / \mathrm{kg}$ ) of 0.32 between load and infusion doses. Because the 40 and $60 \mathrm{mg} / \mathrm{kg} /$ day dose will exceed $9 \mathrm{mg} / \mathrm{kg}$ in the loading period, and no data are available for piglets, future toxicology studies should be performed should those higher doses prove effective.

\section{Agent-specific evaluation metrics for screening in Tier 1}

Tier 1 was the short-term (24-h) evaluation of the CsA dose-response of traumatic braininjured piglets. Treatment efficacy was evaluated using two terminal endpoints, neuropathology and mitochondrial function, in animals sacrificed at $24 \mathrm{~h}$ after treatment was 
initiated. Our rationale for using mitochondrial function, specifically maximal coupled respiration (RCR), as a criterion for positive outcome effectiveness is that previous studies demonstrated that RCR decreases within hours after TBI, remains impaired days later, and its early rescue with mitochondrial protectant CsA correlates with cognitive benefits at 7 days after injury $(11,55,79)$. In infant piglets (3-5 days old) treated with $40 \mathrm{mg} / \mathrm{kg} / \mathrm{day}$ CsA IV divided into two injections post-RNR, reductions in RCR were inhibited and maintained at sham levels (44). Similarly, we selected lesion volume ( $\left.V_{\text {inj }}\right)$ at $24 \mathrm{~h}$ post-TBI as a second positive outcome metric based upon prior studies which demonstrated that after CCI in the piglet brain lesion volume is near maximal around $24 \mathrm{~h}$ after injury (19), and that injured volume was reduced by $42 \%$ in the RNR-injured infant animals treated with $40 \mathrm{mg} / \mathrm{kg} / \mathrm{day}$ CsA IV (44).

When we designed the Tier 1 study as a screening paradigm, we understood that the variability of the underlying condition in genetically heterogeneous subjects would require very large animal group sizes (and prohibitively high costs) to identify effect magnitude and formulate a dose-response curve. Thus, cost considerations restricted our study design, making comparisons of size of effect across doses untenable. Therefore, using clinical trials with multiple "success metrics" as our guide, in our analysis we defined positive outcome "thresholds" for pathology and mitochondrial function, and enhanced our statistical power by combining the results from these two sets of animals by determining a total "positive outcome" rate. Consequently, Tier 1 was designed as a screening paradigm to select promising doses for Tier 2 based on the combination of two metrics. As such, the efficacy analysis is not based upon the absolute treatment effect size (lesion size or mitochondrial function); instead, the analysis is based upon achievement of a clinically significant greater number of "positive outcomes" with treatment compared with untreated injured subjects. The definitions of "clinically significant" alterations to mitochondrial function and neuropathology were determined by pilot series to obtain thresholds prior to evaluation of the experimental data. Based on criteria discussed in the next two sections, each subject was classified as either a "neuropathology lesion volume positive outcome" or "mitochondrial function positive outcome." Efficacy was defined when $\geq 30 \%$ more of the $\mathrm{N}=20$ animals in a CsA dose/time/model group had a combined positive outcome (based upon mitochondrial function and neuro-pathology) compared with injured time/model controls receiving only saline. This is a very ambitious threshold to identify promising therapies as a consequence of budgetary constraints dictating small study cohort sizes.

Because it is postulated that the optimal dose may vary with injury-to-treatment interval and between focal and diffuse injuries, each post-injury start time and each injury type were analyzed independently (four groups: 1-h delay to treatment initiation CCI, 6-h CCI, 1-h RNR, 6-h RNR). For each group, all doses that met the efficacy criteria were eligible to move forward to Tier 2 experiments to determine longer term neurofunctional outcomes. However, the platform was designed so that if multiple doses for a post-injury start time showed efficacy at the end of Tier 1, a maximum of two doses for each start time/injury-type group would move forward to Tier 2, the dose with the highest success rate ("greatest neuroprotection") and the lowest dose that meets the success criterion for demonstrating a successful neuroprotective effect, to potentially limit the side-effect profile. Furthermore, if 
we found a tie for two doses with the highest success rate at an administration time for a given model, we would select the dose that has the highest magnitude of positive outcome. The maximum number of dose-model-treatment time combinations moving forward from Tier 1 to Tier 2 would be 8 ( 2 doses $\times 2$ models $\times 2$ delays to treatment $)$ plus normative controls, a dramatic reduction from the total number across all of the dosing groups studied in Tier 1. This strategy was chosen as a practical way to limit the trial animal and resource utilization and expense.

\section{Pathology threshold for positive outcome}

To judge effectiveness in Tier 1 screening, we had to determine a threshold for each surrogate outcome measure (pathology and mitochondrial energetics) that would be used to designate a subject as showing a likely effect of CsA. We defined a subject classified as showing a clinically significant "positive outcome" for the pathology effectiveness metric as an animal with a reduction in lesion volume that is large compared with the distribution found in the saline-treated (vehicle) animals with the same injury type. To accomplish this, we determined the mean and standard deviation of the lesion volumes in a relevant injurymatched vehicle group (CCI or RNR) generated prospectively prior to the Tier 1 experiments and established a threshold of 0.85 times the standard deviation $\left(0.85^{*} \mathrm{stdev}\right)$ less than this vehicle group mean. Because of small but potentially meaningful differences in the specific CCI devices and the rotational velocities used in the injuries between the first and second years of the Tier 1 study, we determined a pathology threshold that was unique to each year. Therefore, each treated (and vehicle) animal was evaluated against the relevant "positive outcome" threshold to determine pathology positive outcome rates for every dose/ time/model group. Importantly, this bar was more demanding (larger injury reduction required to be considered a "positive outcome") when the injury volume was more variable in our vehicle control group. In fact, this objectively derived pathology threshold established that a reduction in lesion volume of $21 \%-58 \%$ was required for a CsA-treated animal to be considered a positive outcome (across all TBI models, times and years), averaging $25 \%$ in the RNR groups and 50\% in the CCI groups-a dramatic reduction in pathology. The total number of "Pathology positive outcomes" is provided in the Results (Table 2). In short, for each model, defining the threshold for a positive outcome as the mean $+0.85 * \operatorname{stdev}$ of the untreated injured group set the "positive outcome" rate at $20 \%$ of the untreated injured vehicle controls. We found that the treatment groups had positive outcome rates of $0 \%-$ $60 \%$.

\section{Mitochondrial threshold for positive outcome}

For diffuse brain injury, we defined the mitochondrial functional threshold for positive outcome as the RCR measured in isolated mitochondria greater or equal to 5 (after combining results from the cortex and hippocampal regions) based upon our own prior data in the RNR model (44). Historically, a single RCR value is rarely used to define an injury threshold for focal TBI models. Rather, RCR in the ipsilateral injured side is typically compared with a benchmark value, for example, RCR in the contralateral side in the same animal, ipsilateral side in sham controls, or the ipsilateral side in treated injured animals. Moreover, our extensive review of the animal literature on this specific aspect of mitochondrial functional assessment reveals that the RCR on the ipsilateral injured side can 
vary considerably, lab to lab, justifying the use of comparative benchmark ratios for focal brain inju ries, usually the contralateral side in the same animal, thus generating an RCR ratio. We generated a separate set of $(\mathrm{N}=5)$ untreated $\mathrm{CCI}$ piglets to define the positive outcome threshold for the mitochondrial function metric. Not surprisingly, the mean ipsilateral : contralateral RCR ratio of this untreated injured piglet group (mean \pm stdev, 0.55 \pm 0.33 ) was significantly lower than those from $\mathrm{N}=7$ uninjured shams $(1.04 \pm 0.46, P=$ $0.03)$. As with the pathology positive outcome threshold, we used the mean and standard deviation (stdev) of our untreated CCI group to define an objective threshold for a positive outcome as $0.85 \mathrm{stdev}$ greater than the mean untreated value. Specifically, a positive outcome was an RCR ipsilateral/contralateral ratio value $\searrow 0.83$, derived as the mean RCR ratio of untreated injured controls plus 0.85 times the standard deviation, or $0.55+(0.85)$ $(0.33)=0.83$.

Tier 1 studies were conducted over 2 years. In year 2, for the second half of the Tier 1 studies, we took advantage of rapidly evolving technological developments in mitochondrial assessment to provide a more robust evaluation of mitochondrial function. We made three improvements in our measurements of mitochondrial respiration. First, we transitioned from tissue mitochondrial isolation techniques to new protocols developed to utilize tissue homogenates for mitochondrial respiration. Homogenates potentially have several advantages over isolated mitochondria preparations: reduced disruption of intracellular and intercellular signaling pathways (94), preservation of mitochondrial structure and function $(63,64)$, and decreased mitochondria population loss, which may be $>60 \%$ with isolation techniques (62). Thus, homogenates may provide a more relevant and reliable assessment of in vivo function. As a second technological improvement in year 2, we upgraded our traditional Clark-type oxygen electrode (Hansatech Instruments, Norfolk, UK) that measures oxygen consumption in nmols to a more sensitive instrument (Oxygraph-2K, OROBOROS Instruments, Innsbruck, Austria) that measures oxygen consumption in pmol, providing a more consistent and nuanced readout of mitochondrial respiration. Third, we upgraded our respiration protocol by adding the substrate succinate to allow us to measure oxidative phosphorylation via integrated convergent complex I and II electron flow (33), whereas our year 1 standard respiration protocol only utilized NADH-linked substrates (pyruvate + malate).

We therefore generated an additional set of benchmark untreated sham $(\mathrm{N}=20)$ and injured (CCI or RNR N $=20$ ) animals. We confirmed that RCR 5 still distinguished RNR-injured animals from shams, but while the ipsilateral to contralateral ratio of total oxidative phosphorylation was similar after these technological enhancements, the ratio measurement now had less variability. Specifically, the untreated CCI total oxidative phosphorylation ipsilateral to contralateral ratio was $0.61 \pm 0.15$, yielding a positive outcome defined as a ratio $\searrow$.73. In parallel manner to the evaluation of positive outcomes for pathology, CCI animals in year 1 were evaluated against the year 1 threshold, and those in year 2 were evaluated against the year 2 CCI threshold. The total number of "Mitochondrial function positive outcomes" is provided in Table 2. For each model, about $35 \%$ of the vehicle controls met the criteria to be designated as spontaneous "positive outcomes," while the treatment groups had mitochondrial functional positive outcome rates of $30 \%-70 \%$. 


\section{Statistical considerations}

Power calculations were performed to determine the minimal difference that can be detected with the proposed sample size of 20/group in Tier 1 and 30/group in Tier 2. We assume onesided $\alpha=0.05$ and $80 \%$ power; one-sided tests were chosen to identify potentially efficacious strategies. For binary outcomes such as positive outcome rates, Table 1 shows the detectable rate differences, based on Fisher's exact test, for various underlying control rates. The maximum width of (two-sided) 95\% confidence intervals is also shown. For continuous outcomes, such as mean RCR values, the detectable difference is presented as the effect size (Wilcoxon rank-sum test); the width of a 95\% confidence interval is also shown. Based on our previous data (44), we expected the spontaneous positive outcome rate in controls to be approximately $10 \%$, and selected our group sizes accordingly, using $\mathrm{N}=20$ evaluable animals per dose/time/model group for Tier 1 (24-h outcomes).

Prior to formal statistical analysis of outcomes, potential data errors or outliers and necessary variable transformations were examined. Standard descriptive statistics including means, standard deviations and proportions were computed for all measures. Confidence intervals (95\%) were produced for all summary statistics. In Tier 2, exact versions of nonparametric tests were to be used for k-group comparisons, including Wilcoxon and Kruskal-Wallis tests for continuous measures and Fisher's exact or McNemar's tests for proportions. Linear regression was also planned to evaluate associations among secondary endpoints obtained in Tier 2 . The dependent variables will be the primary endpoints from Tier 1 (mitochondrial function and injury volume) and Tier 2 (cognition, balance, actigraphy). Predictor variables will be the potential physio-markers (point-of-care metrics such as microdialysis and serum biomarkers). It is recognized that the large number of simultaneous experiments raises the possibility of inflation of type I error through these future multiple comparisons. This would be carefully considered in the evaluation of all results.

Tier 1 efficacy was defined as having $230 \%$ more of the $\mathrm{N}=20$ animals in a CsA dose/time/ model group with a combined positive outcome (based upon mitochondrial function and neuropathology) compared with injured time/model controls receiving only saline (Agentspecific evaluation metrics for screening in Tier 1). The specific numbers and calculations used to determine positive outcome rate in this study are designed to overcome the inherent limitations of using small sample sizes to estimate the response of a population. Essentially, because of the significant expense of large animal work, statistical approaches must be used to determine true potential or underlying promise from a small number of subjects. In the Results (Tier 1 positive outcome rates), we report that we have a fairly consistent spontaneous "positive outcome" rate of 30\%-35\% in our vehicle control animals because even after mild-to-moderate rapid head rotation or cortical impact, approximately one-third of the animals have modest mitochondrial or pathological damage. Our effectiveness evaluation dictates that for a dose to move on to Tier 2, a treated group must have an underlying "positive outcome" rate of $60 \%-65 \%$, respectively. Statistically, the larger the group one samples, the more likely the group estimates the true or underlying response of a population. For smaller groups, we must consider the errors associated with our uncertainty 
that the results reflect the true or underlying positive outcome rate of the injured animal population.

If we fail to consider the response rate measurement uncertainty in our preclinical study with 20 animals in each of the four dose-time study arms, we would reject any dose that had less than 13 animals (which is $65 \%$ of $\mathrm{N}=20$ ) "above the positive outcome bar" in each group. However, because our measurement of a "positive outcome" rate is only an approximation of the true population response with this small sample size, we would have a $40 \%$ probability of excluding a dose with an inherent positive outcome rate that met our criteria ( $40 \%$ beta error). This $40 \%$ probability of excluding a dose with an underlying response rate that actually met our effectiveness evaluation criteria is a risk of rejecting a successful dose that far exceeds the standard for screening for a promising agent in the pharmaceutical industry (10). Instead, we set our beta error risk at the pharmaceutical industry standard for single-arm randomized clinical trials (10), accepting a 5\% chance of excluding a dose with an inherent positive outcome rate that met our effectiveness evaluation criteria of moving a dose forward if its underlying positive outcome rate $\geq 60 \%-65 \%$. More details are provided in Tier 1 positive outcome rates.

\section{Tier 1 therapy development study design}

Only injured animals were evaluated in Tier 1. Injured animals received either saline vehicle $(0 \mathrm{mg} / \mathrm{kg})$ or CsA treatment at 1 of four different doses $(10,20,40,60 \mathrm{mg} / \mathrm{kg})$ delivered using a bolus followed by a continuous infusion for $24 \mathrm{~h}$ (five doses). Treatment was initiated $1 \mathrm{~h}$ after injury or $6 \mathrm{~h}$ after injury (two timepoints) and extended for $24 \mathrm{~h}$. Both brain injury types were studied: RNR diffuse injury and CCI focal injury. Taken together, 5 doses $\times 2$ timepoints $\times 2$ injury models yields 20 groups. Each of these 20 dose-time-model groups has two outcome measures evaluated $(\mathrm{N}=10$ animals for neuropathology and $\mathrm{N}=$ 10 for mitochondrial function). Because one of our central hypotheses was that mitigation of mitochondrial dysfunction leads to improved neuropathology, our effectiveness evaluation dictated that the success of a dose must be evaluated by combining treatment-associated improvements in mitochondrial function and pathology ( $\mathrm{N}=20$ per treated group). A second central hypothesis is that the dose of CsA may depend on the delay to initiation of treatment and the injury type. Thus, in our study design, analysis plan and effectiveness evaluation, the five doses were clustered together into four single-arm randomized preclinical trials for CsA, one for each of the four start time-model combinations. As mentioned earlier, untreated injured controls were added in year 2. To reduce costs, we assumed untreated injured controls were similar regardless of the delay to saline administration, and we reduced the number of 6-h controls, as shown in Tables 2 and 3. Taken together, the final Tier 1 planned enrollment was $\mathrm{N}=440$ injured piglets.

Piglets ( $\mathrm{N}=440$ females, 4 weeks old) were anesthetized, instrumented and monitored as described previously (44), and buprenorphine $(0.02 \mathrm{mg} / \mathrm{kg}$ IM) was administered for analgesia. An IV with low absorbance tubing was inserted into the cephalic vein, and tunneled subcutaneously to the mid-scapula to an ambulatory infusion pump (3D BT mini infusion, Strategic Applications Inc., Infusion Technologies, Lake Villa, IL, USA), to administer CsA or saline, and to withdraw pre- and 24-h post-injury blood samples for 
serum protein biomarker analysis. Animals were assigned to receive CCI or RNR injury. As mentioned earlier, for CCI, a skull-mounted, spring-loaded blunt indentation device was used to deform approximately $1 \mathrm{~cm}^{3}$ of the cortical rostral gyrus rapidly (4 ms), with a typical lesion volume $\left(V_{\mathrm{inj}}\right)$ of $8 \%$ of the hemisphere or $4 \%$ of the cerebrum (20). For RNR, the snout was secured, and the head rotated $60-70^{\circ}$ rapidly $(12-20 \mathrm{~ms})$ in the sagittal plane without impact (68). Within each injury group, animals were assigned to a CsA post-TBI start time of 1 or $6 \mathrm{~h}$, and randomized to one of the four CsA dose groups (10, 20, 40, 60 $\mathrm{mg} / \mathrm{kg} / \mathrm{day}$ ) or saline. Treatment was administered intravenously according to the dosing schedule described above. Post-injury, animals were monitored until fully recovered from anesthesia and observed until they demonstrated vocalization (without squealing), steady ambulation, no aggression or avoidance behavior, no piloerection and presence of proper feeding, and then returned to the animal housing unit after the initial IV loading dose, with continuation of the infusion delivered in the housing facility. Typically, during recovery, animals initially exhibited purposeless behavior, had poorly coordinated motor function and were unusually lethargic or easily agitated, similar to mild-to-moderate TBI in the child.

After $24 \mathrm{~h}$ of CsA treatment, animals were anesthetized, and a final blood sample was obtained. Subjects were sacrificed, and either fresh brain tissue was collected to determine the mitochondrial function ( $\mathrm{N}=10$ per group), or brains were perfused transcardially with unbuffered formalin to quantify injury volume ( $\left.V_{\text {inj }}\right)$ via histology ( $\mathrm{N}=10$ per group).

For mitochondrial isolation, the brain was exposed, and specimens were removed rapidly after euthanasia. From RNR animals, bilateral $3-5 \mathrm{~mL}$ regions of cortex and hippocampus were removed. From CCI animals, a 2-cm-per-side cube of cortex and underlying white matter centered at the CCI impact site was removed and necrotic tissue was removed, and a mirrored sample from the contralateral side was resected. From the fresh tissue in year 1 of Tier 1, mitochondria were isolated rapidly (88), and RCR (RCR = State 3 divided by State 4) was determined (72). In year 2 , the same regions were homogenized and a serial substrate, uncoupler, inhibitor titration (SUIT) protocol previously used for rodent brain tissue (43) was optimized for the piglet, and maximal coupled phosphorylating respiration capacity via convergent input through complexes I and II $\left(\mathrm{OXPHOS}_{\mathrm{CI}+\mathrm{CII}}\right)$ was determined and normalized by LEAK respiration or State $4_{0}$.

For histology, after perfusion, brains were removed and immersed in fixative. Brains were cut in serial 3-mm coronal sections, photographed for determining total cerebral volume, and examined for gross pathology to document subdural and subarachnoid hemorrhages and surface contusions. Finally, $6-\mu \mathrm{m}$-thick slices from each 3 -mm coronal section were stained with hematoxylin and eosin (H\&E) and $\beta$-APP, and examined by the neuropathologist on our team (CS, blinded to experimental group) who reviewed every field of every slide microscopically. Documented major pathology includes hemorrhages, established infarcts (changes in staining intensity) and ischemic neurons (cell shrinkage and eosinophilia). Axonal injury was detected via $\beta$-APP immunohistochemistry to identify disruption of axonal flow and regions of ischemic axonal injury. The distribution of axonal and neuronal injury was annotated on the digital photographs for each animal $(31,96)$. To quantify the extent of brain injury, the brain periphery of every 3-mm section was traced to determine the brain area (Photoshop, Adobe Systems Incorporated, San Jose, CA, USA). The locations of 
white matter damage, infarct and ischemia were traced in each slice using the same procedure to determine injured brain area. Lesion volume $\left(V_{\text {inj }}\right)$ was defined as total injured area (axonal injury plus infarct/ischemic lesions) divided by total slice area.

\section{Tier 2 study design}

Tier 2 was designed to mimic the clinical care setting, and prospective criteria for effectiveness have been identified, but studies have not been conducted to date. In Tier 2, we plan to evaluate 6-day post-TBI neurofunctional outcome metrics for up to two doses for each model/time group meeting our effectiveness criteria for Tier 1 . The results of treated injured animals will be compared with injured piglets administered vehicle ( $\mathrm{N}=30$ /group), in naïve shams $(\mathrm{N}=12)$, and $\mathrm{CCI}$ and RNR uninjured shams with an intensive care unit (ICU) experience ( $\mathrm{N}=12 /$ group). The most effective CsA treatment-model combinations will then be tested in males to assess for any gender effects and serum processed for CsA concentrations to translate porcine PK to humans using a computational model. Focal and diffuse injuries will be created as in Tier 1. As in Tier 1, treatment will be an IV load followed by continuous infusion, with treatment duration of $24 \mathrm{~h}$. Saline-only treatment of injured animals will be initiated $1 \mathrm{~h}$ post-injury. During the first $24 \mathrm{~h}$ after injury or sham anesthesia, animals will receive continuous neurointensive care monitoring, including CBF, intracranial pressure (ICP), mean arterial pressure (MAP) and $\mathrm{PbtO}_{2}$, as well as IV fluids and respiratory support. We will use a clinical-based protocol to ensure consistent cerebral perfusion pressure (CPP) and ICP management (9) (see flowchart provided in Figure 2). During this 24-h period, serial blood and microdialysis samples will be obtained to measure PK, biomarkers and lactate/pyruvate ratio (LPR) time courses. The biomarkers and LPR are unconfirmed intensive care measures that will be used in a secondary analysis to guide clinical trial design. These serum, microdialysis and physiological biomarkers are not included in the effectiveness criteria; rather, they will be evaluated as informative prognostic markers of injury severity and recovery.

After the injury has been completed and an unconsciousness time was recorded (reaction time post-injury to an interdigital toe pinch), anesthesia will be switched to a continuous midazolam $(0.1-0.6 \mathrm{mg} / \mathrm{kg} / \mathrm{h})$ and fentanyl $(50 \mu \mathrm{g} / \mathrm{kg} / \mathrm{h})$ IV infusion for $24 \mathrm{~h}$ (Day $0-1)$ while being monitored in the NIH-funded state-of-theart Neurointensive Care and Assessment Facility that houses pigs for up to 3 days after TBI with round-the-clock care. Cerebral microdialysis probe (CMA 12 PAS elite $4 \mathrm{~mm}$ probes, CMA, Torshamnsgatan, Sweden), a thermal diffusion CBF probe (Bowman Perfusion Monitor, Hemedex Cambridge, MA, USA) and a brain tissue oxygenation electrode with temperature probe (Licox, Integra Plainsboro Township, NJ, USA) will be inserted into the frontal lobes of the RNR model. In the CCI model, probes will be placed in the ipsilateral frontal lobe. A fiberoptic ICP monitor (Camino, Integra) will also be placed in the frontal lobe. Mean arterial blood pressure will be monitored via a cuff on the forelimb. Cerebral microdialysis samples will be collected at $1,2,4,6,12,18$ and $24 \mathrm{~h}$ post-injury at a flow rate of $10 \mu \mathrm{L} /$ minute using $0.9 \% \mathrm{NaCl}$ as the dialysate. Immediately after collection, samples will be stored at $-70^{\circ} \mathrm{C}$. Levels of lactate and pyruvate will be measured in the dialysate using a microdialysis analyzer (ISCUS-flex, M Dialysis, Stockholm, Sweden). Lactate-pyruvate ratios will be calculated from their respective values. 
During the 24-h ICU period, piglets will receive continuous neurointensive care monitoring (CBF, ICP, MAP, brain tissue oxygenation content), intravenous fluids and respiratory support (if necessary), and isotonic intravenous fluids to maintain normovolemia and normoglycemia and/or vasopressors to support a CPP $\geq 40 \mathrm{mmHg}$ and ICP $\leq 20 \mathrm{mmHg}$, to ensure consistent management across groups, and to provide fidelity between current firsttier clinical practice for acute management of severe TBI in pediatrics $(3,4,41)$ and our translational platform (see flowchart). All animals that required mechanical ventilation will have minute ventilation adjusted to maintain $\mathrm{PaCO}_{2}$ between 35 and $45 \mathrm{mmHg}$ and oxygen titrated to avoid hypoxia $(5,12)$. While intubated, piglets will receive midazolam $(0.6$ $\mathrm{mg} / \mathrm{kg} / \mathrm{h}$ ) and fentanyl (25-100 $\mathrm{mcg} / \mathrm{kg} / \mathrm{h})$ infusions for sedation and analgesia, respectively, the most common sedation regimen used in human pediatric critical care. Because of the indwelling cerebral monitors, all piglets will receive antibiotics (cefazolin, $25 \mathrm{mg} / \mathrm{kg}$, every $8 \mathrm{~h}$ ) during the 24-h stay in the ICU.

During this 24-h ICU period, 12 serial blood samples will be removed for CsA concentration determination from some of the animals ( $\mathrm{N}=16$ /group) in each injured study group using low absorbance tubing and syringes. Specifically, eight pigs from each group will undergo the following 12-sample PK sampling strategy: pre loading dose, immediately post loading dose, and then $0.5,1.5,2.5,4,6,9,13,17$ and $21 \mathrm{~h}$ after the start of the infusion and immediately prior to end of infusion. Eight pigs from each group will undergo the following complementary 12-sample PK sampling strategy: pre loading dose, immediately post loading dose, and then $0.5,1.5,2,3,5,7,11,15$ and $19 \mathrm{~h}$ after the start of the infusion and immediately prior to end of infusion. Serial blood samples will be obtained from the others $(\mathrm{N}=12$ /group) and all uninjured groups to measure biomarkers at four timepoints: preinjury, and at 8 and $24 \mathrm{~h}$, and 6 days post-injury. The PK and pharmacodynamic data will be used to determine optimal porcine CsA exposure, and we will determine if LPR and biomarker samples correlate with cognitive outcomes in a secondary analysis.

After $24 \mathrm{~h}$, animals will be weaned from support. The same recovery practice used for our 24-h survival studies (in Tier 1) will be implemented for the recovery process of this group. The animals will be returned to the animal care unit when the following criteria for stabilization are met: vocalization without squealing, steady ambulation, no aggression or avoidance behavior, no piloerection, and presence of proper feeding/drinking. The animals will be allowed to recover for the remainder of the day (Day 1). Any animal not meeting these criteria by the end of Day 1 will be sacrificed and excluded from analysis. Upon successful recovery, the animals will continue on to participate in post-injury neurofunctional studies on Days 1-6 post-TBI, as described previously $(40,89,90)$.

To demonstrate neurofunctional efficacy, neurofunctional assessments will be performed for 6 days post-injury. To move forward to consideration for clinical trial, a dose must have a statistically significant improvement in at least one neurofunctional metric compared with the similarly injured group receiving vehicle. Only fully evaluable treated and vehicleadministered subjects from the RNR and CCI injuries (30/group) will be considered, and performance metrics may vary by the injury model, because we find that the distribution of brain injury affects functional outcomes (40). If an effective dose for at least one injury type (diffuse RNR or focal CCI) with significant neurofunctional improvement post-injury in 
female piglets is identified, that dose will be evaluated for one injury model in male piglets to identify any sex-related therapeutic differences.

\section{Record keeping methods}

To facilitate translation from preclinical studies to investigational new drug application submission for a future clinical trial, we developed drug accountability reporting forms and a patient record system to provide easy translation to human trials. In addition, all data entry forms are in a secure "cloud"-based application (REDCap system, Research Electronic Data Capture) to facilitate the multisite data entry (University of Pennsylvania, Massachusetts General Hospital and Edinburgh University), with appropriate backup and user edit logging time stamps. Furthermore, for each of the four groups defined by injury model (RNR or $\mathrm{CCI}$ ) and time ( 1 or $6 \mathrm{~h}$ post-injury), and each of the outcomes (RCR or pathology), a separate randomization schedule was created prospectively. Each schedule used a permuted block design with block sizes of six for the 1-h experiments (which included 10 vehicle controls) and block sizes of five for the 6-h experiments (five vehicle controls). Allowances were made for the replacement of animals that did not complete the experiment and additional controls in year 2. The schedules were created in Excel (Microsoft Corportion, Redmond, WA, USA) and then locked to electronic editing. These rigorous documentation instruments are essential for ease of translation to clinical trials.

\section{RESULTS}

For Tier 1, we studied 497 piglets (Table 2) to achieve the target enrollment of 440 injured subjects in our preclinical trial. We excluded 57 subjects based upon our prospectively defined inclusion/exclusion criteria, including difficulties intubating or placing lines prior to injury, prolonged desaturation ( $>5$ minutes at $<90 \%$ oxygen while breathing room air) after injury, failure to maintain normothermia (between 37 and $39^{\circ} \mathrm{C}$ ), failure to reach milestones for return of spontaneous breathing and return to the husbandry unit by $5 \mathrm{~h}$ post-TBI, and amount of agent dispensed from the infusion pump by sacrifice is $>15 \%$ less than expected. Specifically, animals were excluded secondary to technical issues with the intravenous drug delivery system $(\mathrm{N}=28)$, failure to achieve post-injury recovery benchmarks for return to the husbandry unit $(\mathrm{N}=21)$, and various technical problems pre- or post-injury that precluded adherence to the protocol $(\mathrm{N}=8)$.

\section{Tier 1 positive outcome rates}

Each of the four start time-model combinations was evaluated as a separate single-arm randomized preclinical trial for CsA efficacy. To determine the total positive outcome rate for each dose-time-model combination, the numbers of pathology and mitochondrial function positive outcomes (as defined in Pathology threshold for positive outcome and Mitochondrial threshold for positive outcome) from the $\mathrm{N}=20$ animals studied were added together. The injured vehicle control "spontaneous" total positive outcome rate was highly consistent (30\%-35\%) across all model-time combinations, indicating that about one-third of untreated animals fall above the threshold, similar to the variability in outcomes expected for animal models of mild-to-moderate TBI. Based on our Statistical Considerations, our prospective effectiveness evaluation criteria (Agent-specific evaluation metrics for screening 
in Tier 1) dictated that we designate as candidates all CsA doses with a likelihood of having an underlying total positive outcome rate that is $30 \%$ more than the injured untreated vehicle rate $(30 \%-35 \%)$ for that model-time "trial," or a $60 \%-65 \%$ total positive outcome rate.

Given our sample size and imposing the pharmaceutical industry standard for false-positive and false-negative rates in a traditional single-arm randomized clinical trial (10), this strategy serves to identify the required number of positive outcomes any dose must achieve to be considered as a candidate. The beta error accepted by the industry $(<5 \%)$ ensures that we have a $>95 \%$ chance of identifying all doses with an underlying positive outcome rate required by our 60\%-65\% Evaluation Plan threshold for effectiveness (Agent-specific evaluation metrics for screening in Tier 1).

All positive outcome rates are provided in Table 3. Specifically, using the pharmaceutical industry standard for single-arm randomized clinical trials (10) where beta error is $5 \%$, for Tier 1 group sizes of 20 we accepted any dose with at least 10 positive outcome responses (50\% experimental positive outcome rate) as being consistent with the likelihood of having an underlying rate of at least $65 \%$. For the one model/time combination with a vehicle control positive outcome rate of $30 \%$, our effectiveness evaluation targets an underlying rate of $60 \%$ as an effective dose to continue on to Tier 2, and we accept any dose with nine positive outcomes ( $45 \%$ experimental response rate) as being consistent with the likelihood of having an underlying rate of at least $60 \%$.

No CsA doses in the RNR model with 1-h post-TBI delay start time were effective. One dose $(20 \mathrm{mg} / \mathrm{kg})$ in the RNR 6-h delay start time group and one dose $(60 \mathrm{mg} / \mathrm{kg})$ in the CCI 1-h delay group met our criteria for effectiveness. Three doses (10, 20 and $60 \mathrm{mg} / \mathrm{kg}$ ) met our criteria in the CCI 6-h delay group (shaded in yellow in Table 3). The same data are presented in a graphical format for the four dose-time study arms in Figure 3, with the colored vertical bars indicating total positive outcome rates for each dose, the dark horizontal line indicating the underlying effectiveness evaluation positive outcome rate threshold, the gray zone as the 5\% beta error, and the bright yellow line as the positive outcome rate threshold that must be met to achieve our effectiveness evaluation targets with our small sample size and a 5\% beta error. All doses at or exceeding the yellow line in Figure 3 meet the underlying positive outcome rate prescribed by our effectiveness evaluation plan (Agent-specific evaluation metrics for screening in Tier 1) and are defined as "successful" doses. As can be seen, several doses and administration start times met criteria for being promising and going on to Tier 2 testing for each injury model. Not surprisingly, given the convex parabolic pharmacodynamics for CsA demonstrated in rodent TBI treatment trials $(85,87)$, we often observed a narrow optimal dose range, and we did not observe that a linearly increasing concentration of CsA had increasingly positive therapeutic value.

\section{Tier 1 dose response}

Although this study was underpowered to identify dose-responses in Tier 1, we examined qualitative trends in dose effectiveness in either pathology or mitochondrial function in each injury type. First, we consider trends in dose effectiveness for each injury type, followed by those for each delay to initiation of treatment. Raw data of the injury volume (as a percent of 
total brain volume) (top row, Figure 4) and mitochondrial function (bottom row) are provided (mean $\pm \mathrm{SE}$ ). Arrows indicate which direction is associated with improved outcomes. As in humans, the injury presents with a high degree of variability, even given the same mechanical input (head rotational velocity in RNR and cortical deformation in CCI) as demonstrated by standard error bars in the vehicle no-treatment groups (blue bars) for injured volume (top row) and mitochondrial function (bottom row).

After RNR injury, the $20 \mathrm{mg} / \mathrm{kg}$ dose (green bars, Figure 4) meets the total "positive outcome" effectiveness evaluation rate threshold in the 6-h delay to treatment group by improving both pathology and mitochondrial function. The $60 \mathrm{mg} / \mathrm{kg}$ dose (orange) in the 6$\mathrm{h}$ delay group looks promising but falls below the total positive outcome rate effectiveness evaluation threshold because of larger variability in the mitochondrial response at that dose. After CCI injury, increases in the "positive outcome" response rate with treatment tend to be driven by improvements in lesion volume pathology at higher CsA doses rather than doserelated improvement in mitochondrial function in the severely compromised penumbral region of the cortical lesion.

Given the variability in outcomes in large animal models, it is not surprising to us that the qualitative therapy effect sizes appear relatively small in a large animal, limited-subjectnumber trial design. Given that we can only examine trends because our study is underpowered to examine dose effect sizes, our data obtained in a rigorous study, with two injury models representing the focal and diffuse extremes along the spectrum of TBI, support our hypothesis that the underlying therapeutic mechanism and most effective CsA dose may vary with injury type, as in pheno- and geno-specific cancer therapies.

\section{DISCUSSION}

Our effectiveness evaluation criteria are ambitious: to identify CsA doses that demonstrate clearly demonstrable protection levels for the developing brain. For each start time and model combination, our goal was to identify all effective doses and select two doses to move forward to Tier 2: the minimum effective dose and the dose with the greatest magnitude of neuroprotection. If no doses for a time-model combination demonstrate effectiveness, that time-model combination would be designated as futile and discontinued from further study. Using this strategy, we found that one efficacious CsA dose qualified to move forward to further study in Tier 2 in each of three model-start time trials. Specifically, we find that no CsA doses in the RNR model with 1-h post-TBI delay start time were effective. One dose $(20 \mathrm{mg} / \mathrm{kg})$ in the RNR 6-h delay start time group and one dose $(60 \mathrm{mg} / \mathrm{kg})$ in the CCI 1-h delay group met our criteria and are worthy of study in Tier 2. Three doses (10, 20 and 60 $\mathrm{mg} / \mathrm{kg}$ ) met our criteria in the CCI 6-h delay group. Consistent with our effectiveness evaluation plan, because the lowest dose also had the highest rate of positive outcomes, only that dose $(10 \mathrm{mg} / \mathrm{kg})$ would move forward for study of long-term neurofunctional outcomes. It is interesting to note that lower doses were more effective at longer delays to treatment (6 $\mathrm{h})$ and higher doses were more effective with more acute $(1 \mathrm{~h})$ post-TBI administration. Consistent with other investigators who have reported a parabolic pharmacodynamics in rodent models of TBI $(85,87)$, we identified a narrow optimal dose range. What has been of 
additional interest is that the results suggest that this agent may work differently in the different injury types, paving the way for injury-type-specific neuroprotective strategies.

As we hypothesized, we speculate that these findings may have implications for temporal specificity of therapeutic mechanistic targets. To inform our human trial design, we included two clinically relevant treatment windows ( 1 and 6-h delays to the initiation of 24-h treatment) and found that there are promising doses at both timepoints. We conclude that CsA has a practical, generous and promising therapeutic window. Future studies should investigate further why some doses were effective at $6 \mathrm{~h}$ and not $1 \mathrm{~h}$ post-TBI because this finding could significantly alter some of the usual assumptions about optimal timing of treatment of TBI.

Future preclinical animal studies should investigate the interesting underlying mechanisms associated with injury-specific responses, the role of treatment duration on outcome and a more precise dose-response curve. These studies are outside the scope of this restricted experimental design and our limited funding mechanism, and would require larger group sizes to detect the more subtle changes in outcome that occur with different injury mechanisms and CsA doses. It should be noted that the variability in even the most carefully standardized large animal models almost certainly will limit the effect sizes seen, and this may be a clearer reflection of what would likely occur in a human neuroprotection trial.

This is the first report of a preclinical trial paradigm to evaluate therapeutic effectiveness of any TBI treatment in both isolated focal and diffuse injuries, and our findings show that therapeutic dose level and response mechanisms vary with TBI type. These Tier 1 results support CsA as a potentially promising therapy for both focal and diffuse injuries in the immature brain that warrants further investigation to evaluate neurofunctional effectiveness. With funding for Tier 2 in this translational model, long-term neurofunctional outcomes would be evaluated after a clinically fidelic ICU experience for the promising CsA doses and would include naïve controls for behavior norms, uninjured anesthetized controls who undergo an ICU experience (with potential cognitive and behavioral impact), an injured CCI vehicle group and an injured RNR vehicle control group (total of seven groups, 30/group).

In conclusion, this project is the first immature large animal translational treatment trial for pharmacologic intervention for TBI in children. Its overall structure and milestones were designed specifically to parallel those used in human intervention trials in the pediatric age group. Because TBI is heterogeneous, the trial tests the intervention in two different injury types that bracket the range seen in clinical injury and uses two different times of drug administration and four different doses of drug. We designed our immature large animal model platform as a rigorous preclinical screen for a TBI intervention that overcomes some of the failures attributed to previous rodent trials (45) and specifically included a randomized blinded experimental design, careful attention to statistical considerations, data handling, clinically relevant conditions and efficacy testing in both diffuse and focal TBI. We conclude that our platform, while arduous and detailed, has proven itself as a successful comprehensive preclinical screen to identify potential treatments for use in patients with TBI. 


\section{ACKNOWLEDGMENTS}

The preclinical study was supported by the NIH grant U01NS069545. We thank our NIH partners Drs Ramona Hicks and Linda McGavern for their insightful questions that shaped our study design and their long-standing support for the project. We are grateful for the expert technical assistance, many long hours of participation and deep dedication to the project by Courtney Robertson, Athena Zuppa, Jill Ralston, Ashley Bebee, Kortne Hudick, Sabrina Taylor, Christopher Seufert, Carter Dodge, Marissa Fiorello and Jamie Rose. We appreciate the long, tedious hours contributed by Erica Hummel, Jesi Kim, Peter Chhour, Susie Cha and Rachel Margulies digitizing photographs of injured brain sections to determine lesion volume pathology values.

\section{REFERENCES}

1. Adams JH, Graham DI, Gennarelli TA. Head injury in man and experimental animals: neuropathology. Acta Neurochir Suppl (Wien). 1983; 32:15-30. [PubMed: 6581702]

2. Adams JH, Graham DI, Murray LS, Scott G. Diffuse axonal injury due to nonmissile head injury in humans: an analysis of 45 cases. Ann Neurol. 1982; 12:557-563. [PubMed: 7159059]

3. Adelson PD, Bratton SL, Carney NA, Chesnut RM, du Coudray HE, Goldstein B, et al. Guidelines for the acute medical management of severe traumatic brain injury in infants, children, and adolescents. Chapter 1: introduction. Pediatr Crit Care Med. 2003; 4(Suppl. 3):S2-S4. [PubMed: 12847337]

4. Adelson PD, Bratton SL, Carney NA, Chesnut RM, du Coudray HE, Goldstein B, et al. Guidelines for the acute medical management of severe traumatic brain injury in infants, children, and adolescents. Chapter 17. Critical pathway for the treatment of established intracranial hypertension in pediatric traumatic brain injury. Pediatr Crit Care Med. 2003; 4(Suppl. 3):S65-S67. [PubMed: 12847353]

5. Adelson PD, Clyde B, Kochanek PM, Wisniewski SR, Marion DW, Yonas H. Cerebrovascular response in infants and young children following severe traumatic brain injury: a preliminary report. Pediatr Neurosurg. 1997; 26:200-207. [PubMed: 9436831]

6. Alessandri B, Rice AC, Levasseur J, DeFord M, Hamm RJ, Bullock MR. Cyclosporin A improves brain tissue oxygen consumption and learning/memory performance after lateral fluid percussion injury in rats. J Neurotrauma. 2002; 19:829-841. [PubMed: 12184853]

7. Armstead W, Kurth C. Different cerebral hemodynamic responses following fluid percussion brain injury in the newborn and juvenile pig. J Neurotrauma. 1994; 11:487-497. [PubMed: 7861442]

8. Bates TE, Almeida A, Heales SJ, Clark JB. Postnatal development of the complexes of the electron transport chain in isolated rat brain mitochondria. Dev Neurosci. 1994; 16:321-327. [PubMed: 7768212]

9. Brain Trauma Foundation. Guidelines for the management of severe head injury. J Neurotrauma. 2007; 24(Suppl. 1):S1-S106.

10. Cannistra SA. Phase II trials in journal of clinical oncology. J Clin Oncol. 2009; 27:3073-3076. [PubMed: 19451415]

11. Casey PA, McKenna MC, Fiskum G, Saraswati M, Robertson CL. Early and sustained alterations in cerebral metabolism after traumatic brain injury in immature rats. J Neurotrauma. 2008; 25:603-614. [PubMed: 18454682]

12. Chesnut RM, Marshall LF, Klauber MR, Blunt BA, Baldwin N, Eisenberg HM, et al. The role of secondary brain injury in determining outcome from severe head injury. Journal of Trauma. 1993; 34:216-222. [PubMed: 8459458]

13. Cibulskyte D, Pedersen M, Jakobsen P, Hansen HE, Mortensen J. Pharmacokinetic characterization of a pig model of ciclosporin A nephrotoxicity following intravenous administration. Pharmacol Res. 2007; 56:311-317. [PubMed: 17870611]

14. Doppenberg EM, Choi SC, Bullock R. Clinical trials in traumatic brain injury: lessons for the future. Journal of Neurosurgical Anesthesiology. 2004; 16:87-94. [PubMed: 14676577]

15. Collins FS, Tabak LA. Policy: NIH plans to enhance reproducibility. Nature. 2014; 505:612-613. [PubMed: 24482835]

16. Del Maestro R, McDonald W. Distribution of superoxide dismutase, glutathione peroxidase and catalase in developing rat brain. Mech Ageing Dev. 1987; 41:29-38. [PubMed: 3431167] 
17. Duhaime AC. Large animal models of traumatic injury to the immature brain. Dev Neurosci. 2006; 28:380-387. [PubMed: 16943661]

18. Duhaime AC, Hunter JV, Grate LL, Kim A, Demidenko E, Golden JA, Harris C. Magnetic resonance imaging studies of age-dependent responses to scaled focal brain injury in the piglet. J Neurosurg. 2003; 99:542-548. [PubMed: 12959443]

19. Duhaime AC, Hunter JV, Grate LL, Kim A, Golden J, Demidenko E, Harris C. Magnetic resonance imaging studies of age-dependent responses to scaled focal brain injury in the piglet. J Neurosurg. 2003; 99:542-548. [PubMed: 12959443]

20. Duhaime AC, Margulies SS, Durham SR, O'Rourke MM, Golden JA, Marwaha S, Raghupathi R. Maturation-dependent response of the piglet brain to scaled cortical impact. J Neurosurg. 2000; 93:455-462. [PubMed: 10969944]

21. Duhaime AC, Saykin AJ, McDonald BC, Dodge CP, Eskey CJ, Darcey TM, et al. Functional magnetic resonance imaging of the primary somatosensory cortex in piglets. J Neurosurg. 2006; 104(Suppl. 4):259-264. [PubMed: 16619637]

22. Durham SR, Duhaime AC. Maturation-dependent response of the immature brain to experimental subdural hematoma. J Neurotrauma. 2007; 24:5-14. [PubMed: 17263666]

23. Durham SR, Raghupathi R, Helfaer MA, Marwaha S, Duhaime AC. Age-related differences in acute physiologic response to focal traumatic brain injury in piglets. Pediatr Neurosurg. 2000; 33:76-82. [PubMed: 11070433]

24. Eucker SA, Smith C, Ralston J, Friess S, Margulies SS. Physiological and histopathological responses following closed rotational head injury depend on direction of head motion. Experimental Neurology. 2011; 227(1):79-88. [PubMed: 20875409]

25. Ferrand-Drake M, Zhu C, Gido G, Hansen AJ, Karlsson JO, Bahr BA, et al. Cyclosporin A prevents calpain activation despite increased intracellular calcium concentrations, as well as translocation of apoptosis-inducing factor, cytochrome $\mathrm{c}$ and caspase-3 activation in neurons exposed to transient hypoglycemia. J Neurochem. 2003; 85:1431-1442. [PubMed: 12787063]

26. Finn WF, McCormack AJ, Sullivan BA, Hak LJ, Clark RL. Influence of the rate of infusion on cyclosporine nephrotoxicity in the rat. Ren Fail. 1989; 11:3-15. [PubMed: 2772285]

27. Fisher M, Feuerstein G, Howells DW, Hurn PD, Kent TA, Savitz SI, Lo EH. Update of the stroke therapy academic industry roundtable preclinical recommendations. Stroke. 2009; 40(6):22442250. [PubMed: 19246690]

28. Folbergrova J, Li PA, Uchino H, Smith ML, Siesjo BK. Changes in the bioenergetic state of rat hippocampus during $2.5 \mathrm{~min}$ of ischemia, and prevention of cell damage by cyclosporin A in hyperglycemic subjects. Exp Brain Res. 1997; 114:44-50. [PubMed: 9125450]

29. Friess SH, Ichord RN, Owens K, Ralston J, Rizol R, Overall KL, et al. Neurobehavioral functional deficits following closed head injury in the neonatal pig. Exp Neurol. 2007; 204:234-243. [PubMed: 17174304]

30. Fukui S, Signoretti S, Dunbar JG, Marmarou A. The effect of cyclosporin A on brain edema formation following experimental cortical contusion. Acta Neurochir Suppl. 2003; 86:301-303. [PubMed: 14753457]

31. Gentleman SM, Roberts GW, Gennarelli TA, Maxwell WL, Adams JH, Kerr S, Graham DI. Axonal injury: a universal consequence of fatal closed head injury? Acta Neuropathol (Berl). 1995; 89:537-543. [PubMed: 7676809]

32. Ghafari A, Makhdoomi K, Ahmadpour P, Afshari AT, Fallah MM, Rad PS. Low-dose versus highdose cyclosporine induction protocols in renal transplantation. Transplant Proc. 2007; 39:12191222. [PubMed: 17524937]

33. Gnaiger E. Capacity of oxidative phosphorylation in human skeletal muscle: new perspectives of mitochondrial physiology. Int J Biochem Cell Biol. 2009; 41:1837-1845. [PubMed: 19467914]

34. Graham D, Ford I, Adams J, Doyle D, Lawrence A, McLellan D. Fatal head injury in children. J Clin Pathol. 1989; 42:18-22. [PubMed: 2921340]

35. Graham DI, McIntosh TK, Maxwell WL, Nicoll JA. Recent advances in neurotrauma. J Neuropathol Exp Neurol. 2000; 59:641-651. [PubMed: 10952055]

36. Hall ED, Traystman RJ. Role of animal studies in the design of clinical trials. Front Neurol Neurosci. 2009; 25:10-33. [PubMed: 19478492] 
37. Hansson MJ, Persson T, Friberg H, Keep MF, Rees A, Wieloch T, Elmer E. Powerful cyclosporin inhibition of calcium-induced permeability transition in brain mitochondria. Brain Res. 2003; 960:99-111. [PubMed: 12505662]

38. Hoyert DL, Heron MP, Murphy SL, Kung HC. Deaths: final data for 2003. Natl Vital Stat Rep. 2006; 54:1-120. [PubMed: 16689256]

39. Hutchison JS, Ward RE, Lacroix J, Hebert PC, Barnes MA, Bohn DJ, et al. Hypothermia therapy after traumatic brain injury in children. N Engl J Med. 2008; 358:2447-2456. [PubMed: 18525042]

40. Jaber S, Sullivan S, Margulies SS. Noninvasive metrics for identification of brain injury deficits in piglets. Dev Neuropsychol. 2015; 40(1):34-39. [PubMed: 25649778]

41. Jankowitz BT, Adelson PD. Pediatric traumatic brain injury: past, present and future. Dev Neurosci. 2006; 28:264-275. [PubMed: 16943650]

42. Josan VA, Sgouros S. Early decompressive craniectomy may be effective in the treatment of refractory intracranial hypertension after traumatic brain injury. Childs Nerv Syst. 2006; 22:12681274. [PubMed: 16496158]

43. Karlsson M, Hempel C, Sjovall F, Hansson MJ, Kurtzhals JA, Elmer E. Brain mitochondrial function in a murine model of cerebral malaria and the therapeutic effects of rhEPO. Int $\mathrm{J}$ Biochem Cell Biol. 2013; 45:151-155. [PubMed: 22903021]

44. Kilbaugh TJ, Bhandare S, Lorom DH, Saraswati M, Robertson CL, Margulies SS. Cyclosporin A preserves mitochondrial function after traumatic brain injury in the immature rat and piglet. $\mathrm{J}$ Neurotrauma. 2011; 28:763-774. [PubMed: 21250918]

45. Landis SC, Amara SG, Asadullah K, Austin CP, Blumenstein R, Bradley EW, et al. A call for transparent reporting to optimize the predictive value of preclinical research. Nature. 2012; 490:187-191. [PubMed: 23060188]

46. Levin HS, Mendelsohn D, Lilly MA, Yeakley J, Song J, Scheibel RS, et al. Magnetic resonance imaging in relation to functional outcome of pediatric closed head injury: a test of the OmmayaGennarelli model. Neurosurgery. 1997; 40:432-440. [PubMed: 9055281]

47. Li PA, Kristian T, He QP, Siesjo BK. Cyclosporin A enhances survival, ameliorates brain damage, and prevents secondary mitochondrial dysfunction after a 30-minute period of transient cerebral ischemia. Exp Neurol. 2000; 165:153-163. [PubMed: 10964494]

48. Lin MT, Beal MF. Mitochondrial dysfunction and oxidative stress in neurodegenerative diseases. Nature. 2006; 443:787-795. [PubMed: 17051205]

49. Lum BL, Kaubisch S, Fisher GA, Brown BW, Sikic BI. Effect of high-dose cyclosporine on etoposide pharmacodynamics in a trial to reverse P-glycoprotein (MDR1 gene) mediated drug resistance. Cancer Chemother Pharmacol. 2000; 45:305-311. [PubMed: 10755319]

50. Margulies S, Hicks R, Leaders CTfTW. Combination Therapies for Traumatic Brain InjuryProspective Considerations. J Neurotrauma. 2009; 26(6):925-939. [PubMed: 19331514]

51. Margulies S, Thibault L, Gennarelli T. Physical model simulations of brain injury in the primate. J Biomech. 1990; 23:823-836. [PubMed: 2384494]

52. Marklund N, Bakshi A, Castelbuono DJ, Conte V, McIntosh TK. Evaluation of pharmacological treatment strategies in traumatic brain injury. Curr Pharm Des. 2006; 12:1645-1680. [PubMed: 16729876]

53. Mazzeo AT, Alves OL, Gilman CB, Hayes RL, Tolias C, Niki Kunene K, Ross Bullock M. Brain metabolic and hemodynamic effects of cyclosporin A after human severe traumatic brain injury: a microdialysis study. Acta Neurochir (Wien). 2008; 150:1019-1031. discussion 31. [PubMed: 18781275]

54. Mazzeo AT, Brophy GM, Gilman CB, Alves OL, Robles JR, Hayes RL, et al. Safety and tolerability of cyclosporin a in severe traumatic brain injury patients: results from a prospective randomized trial. J Neurotrauma. 2009; 26:2195-2206. [PubMed: 19621985]

55. Mbye LH, Singh IN, Carrico KM, Saatman KE, Hall ED. Comparative neuroprotective effects of cyclosporin A and NIM811, a nonimmunosuppressive cyclosporin A analog, following traumatic brain injury. J Cereb Blood Flow Metab. 2009; 29:87-97. [PubMed: 18714331] 
56. Wright DW, Yeatts SD, Silbergleit R, Palesch YY, Hertzberg VS, Frankel M, et al. Very early administration of progesterone for acute traumatic brain injury. N Engl J Med. 2014; 371:24572466. [PubMed: 25493974]

57. Missios S, Harris BT, Dodge CP, Simoni MK, Costine BA, Lee YL, et al. Scaled cortical impact in immature swine: effect of age and gender on lesion volume. J Neurotrauma. 2009; 26:1943-1951. [PubMed: 19469691]

58. Moppett IK. Traumatic brain injury: assessment, resuscitation and early management. Br J Anaesth. 2007; 99(1):18-31. [PubMed: 17545555]

59. Morrison B 3rd, Saatman KE, Meaney DF, McIntosh TK. In vitro central nervous system models of mechanically induced trauma: a review. J Neurotrauma. 1998; 15:911-928. [PubMed: 9840765]

60. Narayan RK, Michel ME, Ansell B, Baethmann A, Biegon A, Bracken MB, et al. Clinical trials in head injury. J Neurotrauma. 2002; 19:503-557. [PubMed: 12042091]

61. Patrick PD, Blackman JA, Mabry JL, Buck ML, Gurka MJ, Conaway MR. Dopamine agonist therapy in low-response children following traumatic brain injury. J Child Neurol. 2006; 21:879885. [PubMed: 17005105]

62. Pecinova A, Drahota Z, Nuskova H, Pecina P, Houstek J. Evaluation of basic mitochondrial functions using rat tissue homogenates. Mitochondrion. 2011; 11:722-728. [PubMed: 21664301]

63. Picard M, Taivassalo T, Gouspillou G, Hepple RT. Mitochondria: isolation, structure and function. J Physiol. 2011; 589(Pt 18):4413-4421. [PubMed: 21708903]

64. Picard M, Taivassalo T, Ritchie D, Wright KJ, Thomas MM, Romestaing C, Hepple RT. Mitochondrial structure and function are disrupted by standard isolation methods. PLoS ONE. 2011; 6:e18317. [PubMed: 21512578]

65. Povlishock JT, Katz DI. Update of neuropathology and neurological recovery after traumatic brain injury. J Head Trauma Rehabil. 2005; 20:76-94. [PubMed: 15668572]

66. Prabhakaran P, Reddy AT, Oakes WJ, King WD, Winkler MK, Givens TG. A pilot trial comparing cerebral perfusion pressure-targeted therapy to intracranial pressure-targeted therapy in children with severe traumatic brain injury. J Neurosurg. 2004; 100(5 Suppl. Pediatrics):454-459. [PubMed: 15287454]

67. Raghupathi R. Cell death mechanisms following traumatic brain injury. Brain Pathology. 2004; 14:215-222. [PubMed: 15193035]

68. Raghupathi R, Margulies SS. Traumatic axonal injury after closed head injury in the neonatal pig. J Neurotrauma. 2002; 19:843-853. [PubMed: 12184854]

69. Raghupathi R, Mehr M, Helfaer M, Margulies S. Traumatic axonal injury is exacerbated following repetitive close head injury in the neonatal pig. J Neurotrauma. 2004; 21:307-316. [PubMed: 15115605]

70. Reagan-Shaw S, Nihal M, Ahmad N. Dose translation from animal to human studies revisited. FASEB J. 2008; 22:659-661. [PubMed: 17942826]

71. Roberts I, Schierhout G, Alderson P. Absence of evidence for the effectiveness of five interventions routinely used in the intensive care management of severe head injury: a systematic review [see comments]. J Neurol Neurosurg Psychiatry. 1998; 65:729-733. [PubMed: 9810947]

72. Robertson CL, Saraswati M, Fiskum G. Mitochondrial dysfunction early after traumatic brain injury in immature rats. J Neurochem. 2007; 101:1248-1257. [PubMed: 17403141]

73. Robertson CL, Soane L, Siegel ZT, Fiskum G. The potential role of mitochondria in pediatric traumatic brain injury. Dev Neurosci. 2006; 28:432-446. [PubMed: 16943666]

74. Saatman KE, Duhaime A-C, Bullock R, Maas AIR, Valadka A, Manley GT, et al. Classification of traumatic brain injury for targeted therapies. J Neurotrauma. 2008; 25(July):719-738. [PubMed: 18627252]

75. Saito A, Maier CM, Narasimhan P, Nishi T, Song YS, Yu F, et al. Oxidative stress and neuronal death/survival signaling in cerebral ischemia. Mol Neurobiol. 2005; 31:105-116. [PubMed: 15953815]

76. Schouten JW. Neuroprotection in traumatic brain injury: a complex struggle against the biology of nature. Curr Opin Crit Care. 2007; 13:134-142. [PubMed: 17327733] 
77. Shreiber DI, Smith DH, Meaney DF. Immediate in vivo response of the cortex and the blood-brain barrier following dynamic cortical deformation in the rat. Neurosci Lett. 1999; 259:5-8. [PubMed: 10027542]

78. Signoretti S, Marmarou A, Tavazzi B, Dunbar J, Amorini AM, Lazzarino G, Vagnozzi R. The protective effect of cyclosporin A upon $\mathrm{N}$-acetylaspartate and mitochondrial dysfunction following experimental diffuse traumatic brain injury. J Neurotrauma. 2004; 21:1154-1167. [PubMed: 15453986]

79. Singh IN, Sullivan PG, Deng Y, Mbye LH, Hall ED. Time course of post-traumatic mitochondrial oxidative damage and dysfunction in a mouse model of focal traumatic brain injury: implications for neuroprotective therapy. J Cereb Blood Flow Metab. 2006; 26:1407-1418. [PubMed: $16538231]$

80. Smith DH, Meaney DF, Shull WH. Diffuse axonal injury in head trauma. J Head Trauma Rehabil. 2003; 18:307-316. [PubMed: 16222127]

81. Smith DH, Wolf JA, Lusardi TA, Lee VM, Meaney DF. High tolerance and delayed elastic response of cultured axons to dynamic stretch injury. J Neurosci. 1999; 19:4263-4269. [PubMed: 10341230]

82. Stein SC, Spettell CM. Delayed and progressive brain injury in children and adolescents. Pediatr Neurosurg. 1995; 23:299-304. [PubMed: 8743998]

83. Suehiro E, Ueda Y, Wei EP, Kontos HA, Povlishock JT. Posttraumatic hypothermia followed by slow rewarming protects the cerebral microcirculation. J Neurotrauma. 2003; 20:381-390. [PubMed: 12866817]

84. Sullivan BA, Hak LJ, Finn WF. Cyclosporine nephrotoxicity: studies in laboratory animals. Transplant Proc. 1985; 17(4 Suppl. 1):145-154. [PubMed: 3895655]

85. Sullivan PG, Rabchevsky AG, Hicks RR, Gibson TR, Fletcher-Turner A, Scheff SW. Doseresponse curve and optimal dosing regimen of cyclosporin A after traumatic brain injury in rats. Neuroscience. 2000; 101:289-295. [PubMed: 11074152]

86. Sullivan PG, Rabchevsky AG, Waldmeier PC, Springer JE. Mitochondrial permeability transition in CNS trauma: cause or effect of neuronal cell death? J Neurosci Res. 2005; 79:231-239. [PubMed: 15573402]

87. Sullivan PG, Thompson M, Scheff SW. Continuous infusion of cyclosporin A postinjury significantly ameliorates cortical damage following traumatic brain injury. Exp Neurol. 2000; 161:631-637. [PubMed: 10686082]

88. Sullivan PG, Thompson MB, Scheff SW. Cyclosporin A attenuates acute mitochondrial dysfunction following traumatic brain injury. Exp Neurol. 1999; 160:226-234. [PubMed: 10630207]

89. Sullivan S, Friess SH, Ralston J, Smith C, Propert KJ, Rapp PE, Margulies SS. Behavioral deficits and axonal injury persistence after rotational head injury are direction dependent. J Neurotrauma. 2013; 30:538-545. [PubMed: 23216054]

90. Sullivan S, Friess SH, Ralston J, Smith C, Propert KJ, Rapp PE, Margulies SS. Improved behavior, motor, and cognition assessments in neonatal piglets. J Neurotrauma. 2013; 30:1770-1779. [PubMed: 23758416]

91. Thompson HJ, Lifshitz J, Marklund N, Grady MS, Graham DI, Hovda DA, McIntosh TK. Lateral fluid percussion brain injury: a 15-year review and evaluation. J Neurotrauma. 2005; 22:42-75. [PubMed: 15665602]

92. Tsao PW, Ito S, Wong PY, Radde IC, Bryson S, Young DS, et al. Pharmacodynamics and pharmacokinetics of cyclosporin A in the newborn pig. Dev Pharmacol Ther. 1992; 18:20-25. [PubMed: 1483359]

93. Turner MS. Early use of intrathecal baclofen in brain injury in pediatric patients. Acta Neurochir Suppl. 2003; 87:81-83. [PubMed: 14518529]

94. Verma G, Datta M. The critical role of JNK in the ER-mitochondrial crosstalk during apoptotic cell death. J Cell Physiol. 2012; 227:1791-1795. [PubMed: 21732347]

95. Werner C, Engelhard K. Pathophysiology of traumatic brain injury. Br J Anaesth. 2007; 99:4-9. [PubMed: 17573392] 
96. Williams S, Raghupathi R, MacKinnon MA, McIntosh TK, Saatman KE, Graham DI. In situ DNA fragmentation occurs in white matter up to 12 months after head injury in man. Acta Neuropathol (Berl). 2001; 102:581-590. [PubMed: 11761718]

97. Yi JH, Hazell AS. Excitotoxic mechanisms and the role of astrocytic glutamate transporters in traumatic brain injury. Neurochem Int. 2006; 48:394-403. [PubMed: 16473439] 


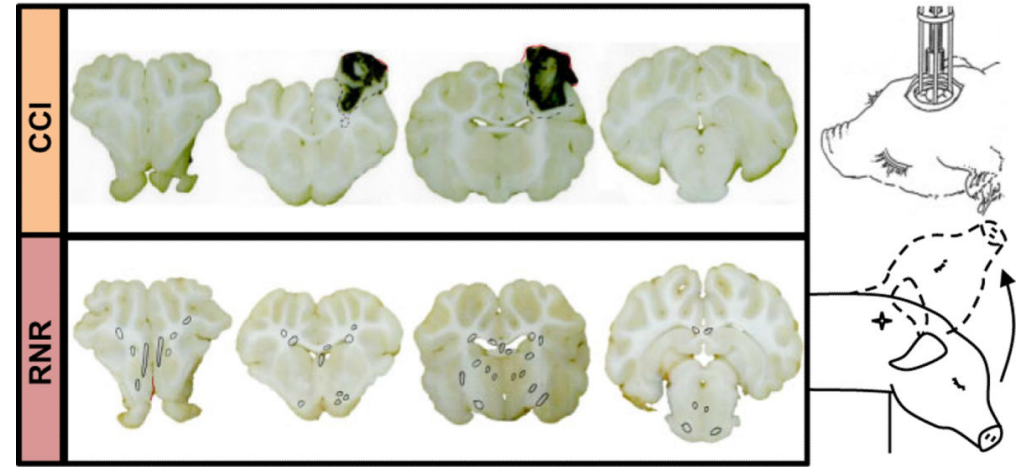

Figure 1.

Schematic of two head injury models. Focal injuries produced by controlled cortical impact (CCI, top) and diffuse brain injuries produced by a sagittal rapid nonimpact rotation (RNR) of the head (bottom). Representative coronal sections shown with regions of axonal injury indicated with circles. 


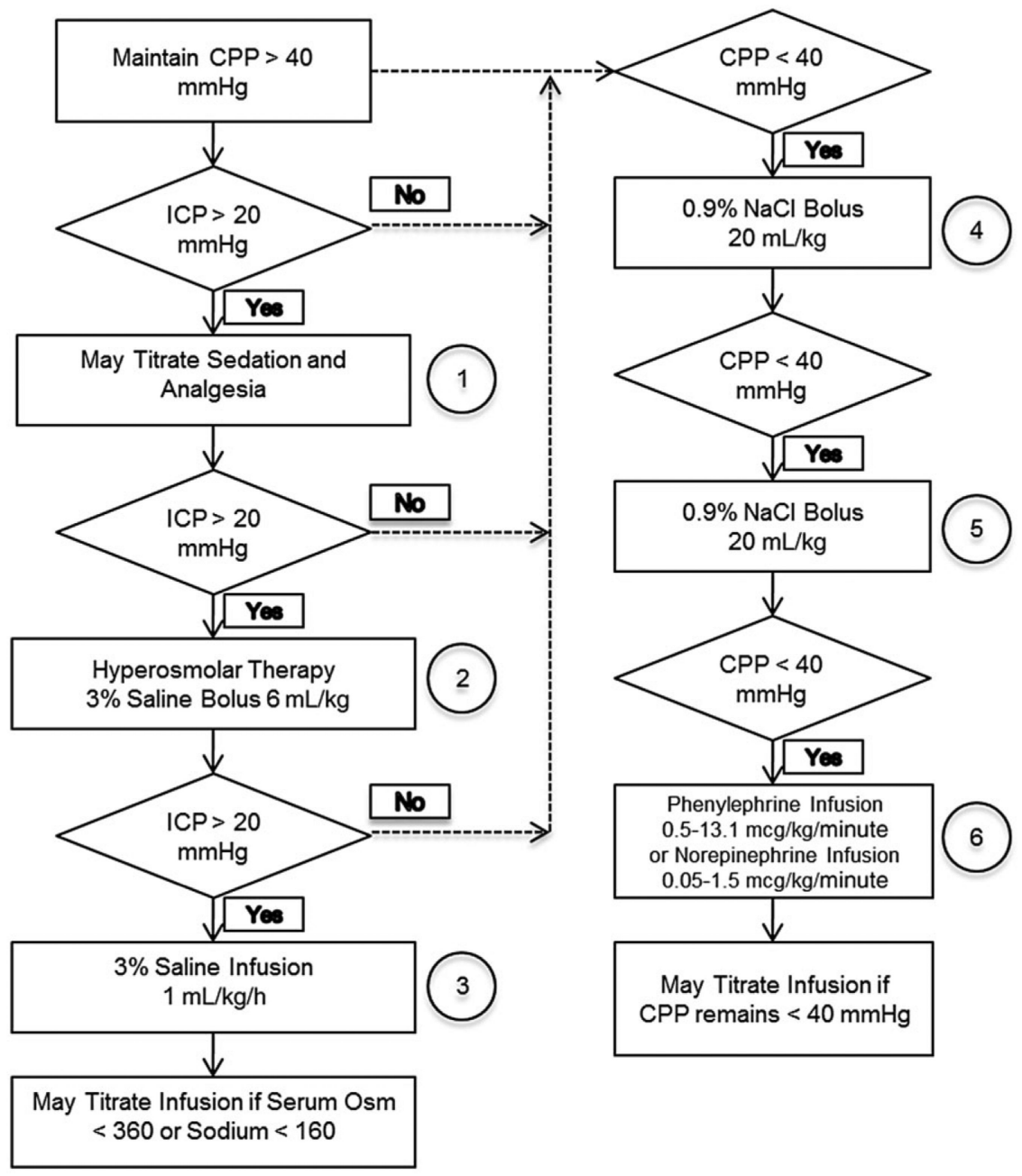

Figure 2.

Tier 2 porcine intensive care cerebral perfusion pressure (CPP) management strategy. 

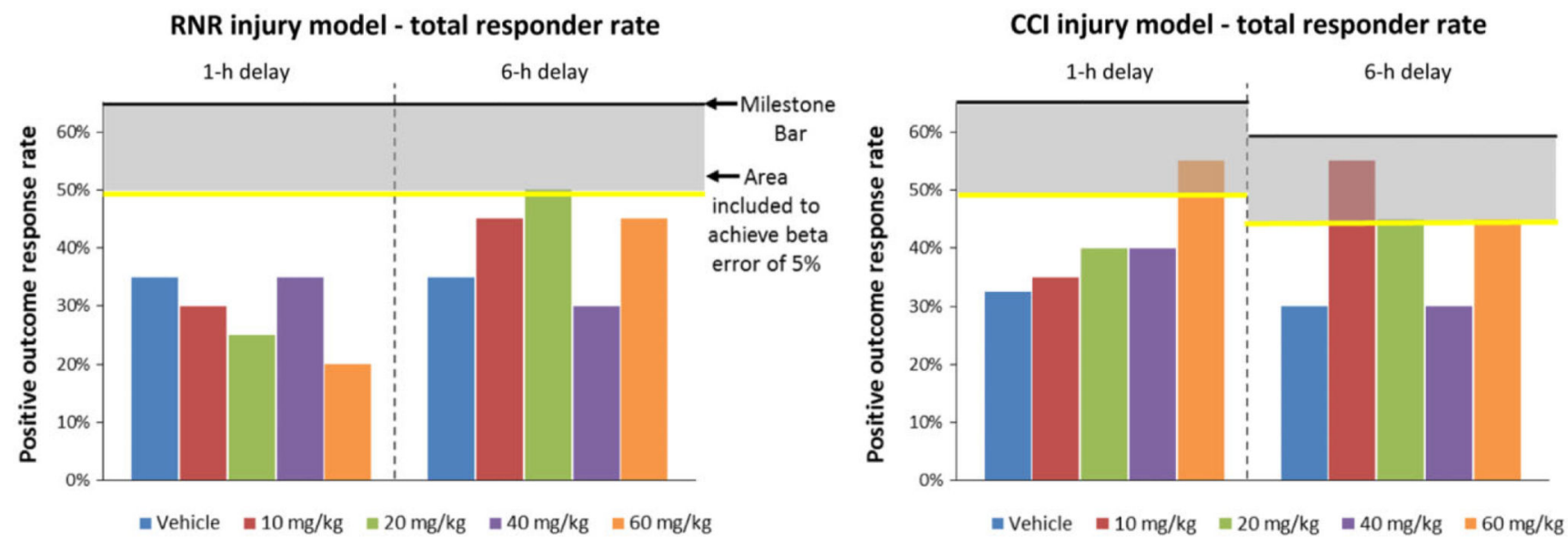

Figure 3.

Tier 1 overall positive outcome rates. Colored vertical bars indicating total positive outcome response rates for each dose, the dark horizontal line indicating the underlying effectiveness evaluation positive outcome rate threshold, the gray zone as the 5\% beta error, and the bright yellow line as the response rate threshold that must be met to achieve our effectiveness evaluation targets with our small sample size and a 5\% beta error. All doses at or exceeding the yellow line meet the underlying positive outcome rate prescribed by our effectiveness evaluation plan, and are defined as "successful" doses. 

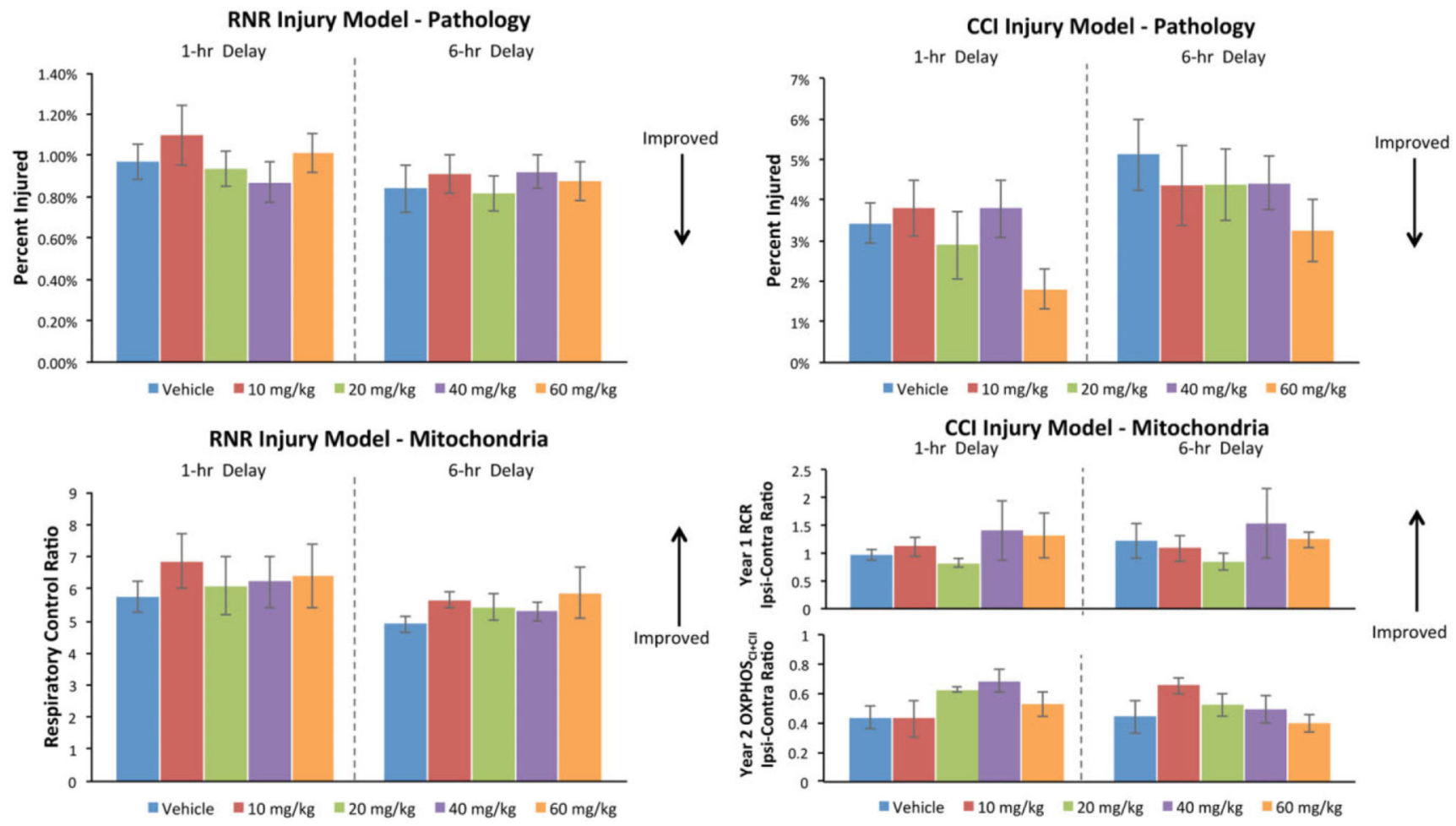

Figure 4.

Tier 1 CsA dose-response findings. 


\section{Table 1}

Group size determination and power considerations.

\begin{tabular}{lrcccccc}
\hline n/group & \multicolumn{3}{l}{ Positive outcome rates } & & \multicolumn{2}{c}{ Continuous measures } \\
\cline { 2 - 5 } \cline { 2 - 4 } & Controls & Treated & Difference & $\mathbf{9 5 \%}$ CI & & Effect size & $\mathbf{9 5 \%}$ CI \\
\hline Total $\mathrm{n}=20$ & $1.0 \%$ & $32.7 \%$ & $31.7 \%$ & & & \\
& $10.0 \%$ & $47.9 \%$ & $37.9 \%$ & $\pm 22.8 \%$ & $0.82 \sigma$ & $\pm 0.47 \sigma$ \\
& $20.0 \%$ & $62.5 \%$ & $42.5 \%$ & & & \\
\hline
\end{tabular}




\section{Table 2}

Tier 1 subjects.

\begin{tabular}{lrrr}
\hline & Pathology & Mitochondria & Excluded \\
\hline 1-h RNR & 60 & 60 & 21 \\
6-h RNR & 50 & 50 & 12 \\
1-h CCI & 60 & 60 & 17 \\
6-h CCI & 50 & 50 & 7 \\
Total & 220 & 220 & 57 \\
\hline
\end{tabular}

Abbreviations: $\mathrm{RNR}=$ rapid nonimpact rotation; $\mathrm{CCI}=$ controlled cortical impact 


\section{Table 3}

Tier 1 overall positive outcome rates. Dose groups that achieve the required positive outcome rate to be considered promising as defined by our Tier 1 effectiveness evaluation plan are shaded in yellow.

\begin{tabular}{|c|c|c|c|c|c|c|}
\hline Group & Outcome & (Vehicle) $0 \mathrm{mg} / \mathrm{kg} / \mathrm{day}$ & $10 \mathrm{mg} / \mathrm{kg} / \mathrm{day}$ & $20 \mathrm{mg} / \mathrm{kg} / \mathrm{day}$ & $40 \mathrm{mg} / \mathrm{kg} / \mathrm{day}$ & $60 \mathrm{mg} / \mathrm{kg} / \mathrm{day}$ \\
\hline \multirow[t]{3}{*}{ 1-h RNR } & Pathology & $4 / 20$ & $0 / 10$ & $1 / 10$ & $2 / 10$ & $0 / 10$ \\
\hline & Mitochondria & $10 / 20$ & $6 / 10$ & $4 / 10$ & $5 / 10$ & $4 / 10$ \\
\hline & Total positive outcome rate & $14 / 40(35 \%)$ & $6 / 20(30 \%)$ & $5 / 20(25 \%)$ & $7 / 20(35 \%)$ & $4 / 20(20 \%)$ \\
\hline \multirow[t]{3}{*}{ 6-h RNR } & Pathology & $2 / 10$ & $2 / 10$ & $4 / 10$ & $1 / 10$ & $3 / 10$ \\
\hline & Mitochondria & $5 / 10$ & $7 / 10$ & $6 / 10$ & $5 / 10$ & $6 / 10$ \\
\hline & Total positive outcome rate & $7 / 20(35 \%)$ & $9 / 20(45 \%)$ & $10 / 20(50 \%)$ & $6 / 20(30 \%)$ & $9 / 20(45 \%)$ \\
\hline \multirow[t]{3}{*}{ 1-h CCI } & Pathology & $4 / 20$ & $1 / 10$ & $4 / 10$ & $2 / 10$ & $6 / 10$ \\
\hline & Mitochondria & $9 / 20$ & $6 / 10$ & $4 / 10$ & $6 / 10$ & $5 / 10$ \\
\hline & Total positive outcome rate & $13 / 40(33 \%)$ & $7 / 20(35 \%)$ & $8 / 20(40 \%)$ & $8 / 20(40 \%)$ & $11 / 20(55 \%)$ \\
\hline \multirow[t]{3}{*}{ 6-h CCI } & Pathology & $2 / 10$ & $4 / 10$ & $5 / 10$ & $3 / 10$ & $4 / 10$ \\
\hline & Mitochondria & $5 / 10$ & $7 / 10$ & $4 / 10$ & $3 / 10$ & $5 / 10$ \\
\hline & Total positive outcome rate & $6 / 20(30 \%)$ & $11 / 20(55 \%)$ & $9 / 20(45 \%)$ & $6 / 20(30 \%)$ & $9 / 20(45 \%)$ \\
\hline
\end{tabular}

Abbreviations: $\mathrm{RNR}=$ rapid nonimpact rotation $\mathrm{CCI}=$ controlled cortical impact. 